\title{
Fluorescence Imaging of siRNA Delivery by Peptide Nucleic Acid-based Probe
}

\author{
Takaya Sato, ${ }^{*}$ Yusuke SAto, ${ }^{* \dagger}$ Kenta IwaI,** Shusuke Kuge, ${ }^{* *}$ Norio Teramae,* and \\ Seiichi NISHIZAWA ${ }^{* \dagger}$ \\ *Department of Chemistry, Graduate School of Science, Tohoku University, Aoba, Sendai 980-8578, Japan \\ **Department of Microbiology, Tohoku Pharmaceutical University, Aoba, Sendai 981-8558, Japan
}

\begin{abstract}
We report on the use of a peptide nucleic acid (PNA)-based fluorescent probe for the analysis of siRNA delivery to living cells. The probe, Py-AA-TO, possesses thiazole orange (TO) and pyrene moieties in the C-and N-termini of PNA, and can function as a light-up probe capable of selective binding to $3^{\prime}$-overhanging nucleotides of target siRNAs. The affinitylabeling of the siRNAs with Py-AA-TO facilitates fluorescence imaging of cellular uptake of polymer-based carriers encapsulating the siRNAs (polyplexes) through endocytosis and subsequent sequestration into lysosome. In addition, flow cytometric measurements reveal that the monitoring of Py-AA-TO fluorescence inside the cells is successfully applicable to the analysis of the polyplex disassembly. These promising functions of Py-AA-TO are presented and discussed as a basis for the design of molecular probes for fluorescent imaging and quantitative analysis of the siRNA delivery process.
\end{abstract}

Keywords Small interfering RNA (siRNA), RNA interference (RNAi), peptide nucleic acid, thiazole orange, fluorescence microscopy, siRNA delivery, delivery carrier, affinity-labeling

(Received December 15, 2014; Accepted January 19, 2015; Published April 10, 2015)

\section{Introduction}

Since the discovery of RNA interference (RNAi) induced by small interfering RNAs (siRNAs), ${ }^{1}$ siRNA has been extensively used as a powerful tool for the study of gene function. In the RNAi machinery, exogenous delivery of synthetic siRNAs into cells results in efficient degradation of complementary mRNAs in a sequence-specific manner. ${ }^{1}$ Thus, siRNA is expected to become a class of next generation drugs targeting various diseases such as cancers ${ }^{2,3}$ and viral infection. ${ }^{4,5}$ Despite its therapeutic potential, intrinsic instability of siRNA against nucleases in biological media and impermeability into cell membranes significantly hinder practical implementation. ${ }^{6,7}$ In order to address these problems, much attention has been paid to the development of carrier molecules, especially non-viral carriers such as lipids and polymers. ${ }^{89}$ For the design of carriers to achieve effective and safe gene silencing, it is of great importance to carefully assess the efficiency of cellular uptake, siRNA releasing and gene knockdown. In addition, it should be also beneficial to decipher at a molecular level the mechanisms of siRNA delivery processes for further development of various RNA-based therapeutics. ${ }^{10}$

In this context, we have recently developed a new class of fluorescent probes that are applicable to fluorescence imagingbased analysis of siRNAs in living cells. Our probes are composed of peptide nucleic acids (PNAs) for targeting

$\dagger$ To whom correspondence should be addressed.

E-mail: satoyuu@m.tohoku.ac.jp (Y. S.); nishi@m.tohoku.ac.jp (S. N.) 3'-overhanging nucleotides uniquely found in siRNAs (Fig. 1). PNAs were further conjugated with thiazole orange (TO) and pyrene to their $\mathrm{C}$ - and $\mathrm{N}$-termini, respectively, which produced the light-up fluorescent probe (Fig. 1B, Py-AA-TO). Py-AA-TO can selectively bind to target siRNAs possessing 2-nt overhanging nucleotides that are complementary to PNA moieties in the probe, and produce a light-up response upon intercalation of the TO moiety into the double-stranded regions near overhangs. ${ }^{11}$ We also demonstrated that Py-AA-TO can function as an affinity-labeling agent of siRNAs for applying to delivery analysis of natural siRNAs without covalent labeling of fluorophores. It is characteristic of Py-AA-TO to selectively visualize siRNAs encapsulated in polymer-based nanocarriers, which then enabled accurate analysis of the delivery process of siRNA-carrier nanocomplexes into living cells. Importantly, by virtue of non-covalent binding of Py-AA-TO with selectivity for target siRNAs, our probe does not compromise the siRNA silencing activity, which is one of the major advantages compared to traditional fluorophore labeling of siRNAs. ${ }^{12}$

In this work, we report on the detailed ability of Py-AA-TO as an affinity-labeling agent for fluorescence imaging of the siRNA delivery process by transfection (Fig. 1A). First, we investigated the effect of Py-AA-TO affinity-labeling on the physicochemical parameters of siRNA-containing polymerbased carrier nanocomplexes (polyplexes) used for the delivery process. Cellular uptake and the intracellular behaviors of the polyplexes were monitored by fluorescence imaging of living HeLa cells, in combination with costaining of nuclei and lysosome. Moreover, these processes and subsequent disassembly of the polyplexes were quantitatively evaluated by flow cytometric measurements. These results were discussed as 
(A)

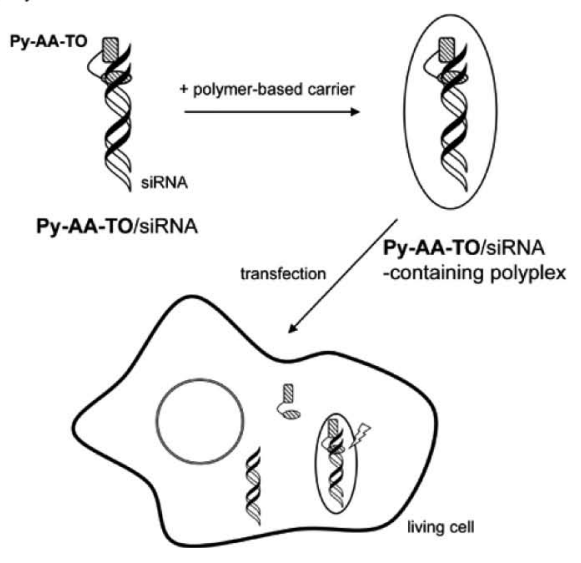

(B)

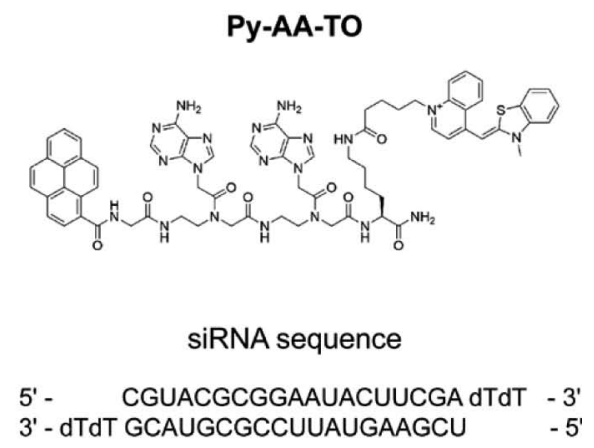

Fig. 1 (A) Schematic illustration for transfection of siRNAs affinity-labeled with Py-AA-TO (PyAA-TO/siRNA) by delivery carriers. Py-AA-TO/siRNA complexes are incubated with polymer-based carriers, and the resulting polyplexes are taken up by living cells. Intracellular polyplexes are subsequently detected by a light-up fluorescence from the TO moiety of Py-AA-TO bound to siRNAs. (B) Chemical structure of Py-AA-TO and siRNA sequence used in this study (siGL2, firefly luciferase GL2-specific sequence).

a basis for the design of molecular probes for the imaging and quantitative analysis of the siRNA delivery process.

\section{Experimental}

\section{Reagents and chemicals}

siGL2 and Alexa647-modified siGL2 (5'-CGU ACG CGG AAU ACU UCG A dTdT- $3^{\prime} / 3^{\prime}-\mathrm{dTdT}$ G CAU GCG CC (Alexa647-U) UAU GAA GCU-5') were custom synthesized and HPLC purified (>99\%) by Sigma-Genosys (Hokkaido, Japan) and GeneDesign, Inc. (Osaka, Japan), respectively. JetPRIME ${ }^{\mathrm{TM}}$ was purchased from Polyplus transfection (Illkirch, France). Dynasore hydrate was purchased from Sigma-Aldrich (Tokyo, Japan). Lipofectamine 2000 and Hoechst 33342 were purchased from Invitrogen (Carlsbad, CA). A dual-luciferase reporter assay system was purchased from Promega (Tokyo, Japan). The other reagents were commercially available analytical grade and were used without further purification.

The concentration of RNAs was determined from the absorbance at $260 \mathrm{~nm}$ at $85^{\circ} \mathrm{C}$ using the molar extinction coefficient provided by the manufacturers. Water was deionized $(\geq 18.0 \mathrm{M} \Omega \mathrm{cm}$ specific resistance) by an Elix $5 \mathrm{UV}$ water purification system and a Milli-Q Synthesis A10 system (Millipore Corp., Bedford, MA), followed by filtration through a BioPak filter (Millipore Corp.) in order to remove RNase. Before all measurements, the sample solutions containing siRNAs were annealed to assure the formation of the siRNA duplexes.

\section{Size and zeta potential measurements}

Polyplexes containing siGL2 or Py-AA-TO/siGL2 were prepared in $20 \mathrm{mM}$ HEPES buffer ( $\mathrm{pH} 7.0$ ) according to the previous report. ${ }^{11}$ Just prior to measurement, the polyplex solutions were diluted 1:10 with HEPES buffer. Final concentration of siRNA or Py-AA-TO/siGL2 was set to $200 \mathrm{nM}$. The samples were measured in a folded capillary cell (DTS1070) with laser light scattering using a Zetasizer Nano ZS with backscatter detection (Malvern Instruments, Worcestershire, UK) at $20^{\circ} \mathrm{C}$. For the determination of the physicochemical parameters, the viscosity and refractive index of the polyplexcontaining solutions were set to $0.89 \mathrm{nPa} \cdot \mathrm{s}$ and 1.330 , respectively. The measurements were performed in triplicate.

\section{Fluorescence microscopy}

The day prior to transfection, HeLa cells were seeded in an 8-well LabTek chamber slide (Sigma-Aldrich, Tokyo, Japan) at a density of $2.5 \times 10^{4}$ cells/well. The polyplex solution was added to the cell culture medium (DMEM, Dulbecco's modified Eagle medium) supplemented with $10 \%$ fetal bovin serum (FBS) at a final Py-AA-TO/siRNA concentration of $200 \mathrm{nM}$ in the polyplexes. The cells treated with the polyplexes were washed with PBS(-) buffer and then imaged in HBSS(-) buffer by the DeltaVision Elite microscopy systems (GE Healthcare Japan, Tokyo, Japan). Costaining of lysosome was done using the Cell Navigator Lysosome Staining Kit (AAT Bioquest, Sunnyvale, CA) according to the manufacturer's instructions. The following filter sets were used: DAPI filter set (Ex 390/18; Em 435/48) for Hoechst 33342; FITC filter set (Ex 475/28; Em 545/48) for Py-AA-TO; TRITC filter set (Ex 545/27; Em 597/45) for lysosome-selective fluorescent probe. The obtained images were deconvolved using softWoRx software.

Time-lapse imaging was performed in a sealed chamber at $37^{\circ} \mathrm{C}$ under humidity with $5 \% \mathrm{CO}_{2}$. In order to minimize the phototoxicity and photobleaching, reduced incident light power and longer exposure time were applied. ${ }^{13}$ Images were captured in phenol-red free DMEM supplemented with $8 \%$ FBS at indicated time points in the images.

\section{Flow cytometry}

Cellular uptake and the disassembly of the polyplexes were evaluated by MoFlo XDP (Beckman Coulter). Briefly, $1.0 \times$ $10^{5} \mathrm{HeLa}$ cells in culture dish were transfected with $300 \mathrm{nM}$ Py-AA-TO/siGL2 or $300 \mathrm{nM}$ Alexa647-siGL2-containing polyplexes and incubated for 1 to $20 \mathrm{~h}$. In the case of long incubation time ( 8 and $20 \mathrm{~h}$ ), cells were washed with PBS(-) three times and then the culture medium was replaced at $4 \mathrm{~h}$ post-transfection to remove any polyplexes that were not taken up by cells. Before flow cytometric measurements, HeLa cells were washed with $\operatorname{PBS}(-)$, tripsinized, and suspended in 
Table 1 DLS diameter and zeta potential of polyplexes containing siGL2 or Py-AA-TO/siGL2

\begin{tabular}{lcc}
\hline & Diameter/nm & Zeta potential/mV \\
\hline siGL2 & $227 \pm 24.7$ & $14.4 \pm 0.544$ \\
Py-AA-TO/siGL2 & $238 \pm 23.2$ & $13.0 \pm 0.736$ \\
\hline
\end{tabular}

HBSS(-) buffer. The following filter sets were used for fluorescence detection: Ex 488, Em 529/28 for Py-AA-TO; Ex 642, Em 670/30 for Alexa647. The percentages of cells that took up the polyplexes were determined by the number of cells in which Py-AA-TO or Alexa647 fluorescence was detected.

\section{Statistical analysis}

The measurements of siRNA silencing activity were repeated three times. All data were expressed as means \pm SD. Statistical analysis was performed using one-way analysis of variance (ANOVA). The probability value $P<0.05$ was considered to be statistically significant.

\section{Results and Discussion}

First, we examined the effect of affinity-labeling of siRNAs with Py-AA-TO on the size and zeta potential of the polyplexes (Table 1), since these physicochemical parameters significantly affect the efficiency of cellular uptake as well as pharmacokinetics. ${ }^{8} 14-16$ From DLS measurements, the particle size of Py-AA-TO/siGL2-containing polyplexes was estimated as $238 \pm 23.2 \mathrm{~nm}$. This value was found to be almost comparable to that of polyplexes without Py-AA-TO (227 \pm $24.7 \mathrm{~nm}$ ). Also, zeta potentials of the polyplexes were found to be little affected in the presence of Py-AA-TO. Both polyplexes exhibited a positive zeta potential of about $+13-15 \mathrm{mV}$, which indicates a complete condensation of siGL2 in these polyplexes. These results suggest that physicochemical parameters of siRNA-containing polyplexes remained almost unchanged by affinity-labeling with Py-AA-TO under the experimental conditions used in this study.

We then investigated cellular uptake and subsequent internalization process of Py-AA-TO/siGL2-containing polyplex by fluorescence microscopy. Figure 2A shows the fluorescence images of $\mathrm{HeLa}$ cells after incubation with Py-AA-TO/siGL2-containing polyplexes for $4 \mathrm{~h}$. A globular, punctate fluorescence of Py-AA-TO was observed in cytoplasm (Fig. 2A, left), and the fluorescence was highly likely from Py-AA-TO bound to siGL2 in the polyplexes. The observed pattern indicates endocytosis-mediated entry of the polyplexes to the cells and subsequent intracellular compartmentalization. ${ }^{17,18}$ The former issue was clarified by the examination of cellular uptake in the presence of Dynasore. Dynasore is an inhibitor of the GTPase activity of dynamins required for membrane fission in clathrin-mediated endocytosis and other endocytic pathways. ${ }^{19,20}$ Indeed, treatment of $80 \mu \mathrm{M}$ Dynasore was found to significantly reduce the uptake of the polyplexes (Fig. 2A, right). This also resulted in the reduction in silencing activity of siGL2 by $40 \%$ (Fig. 2B). On the other hand, the latter issue, intracellular compartmentalization, can be supported by costaining experiments with a lysosome-selective probe. Figure 3 shows fluorescence images of HeLa cells at $4 \mathrm{~h}$ posttransfection of Py-AA-TO/siGL2-containing polyplexes in combination with lysosome staining. Green and red fluorescence represent the polyplex and lysosome, respectively. Apparently,
(A)
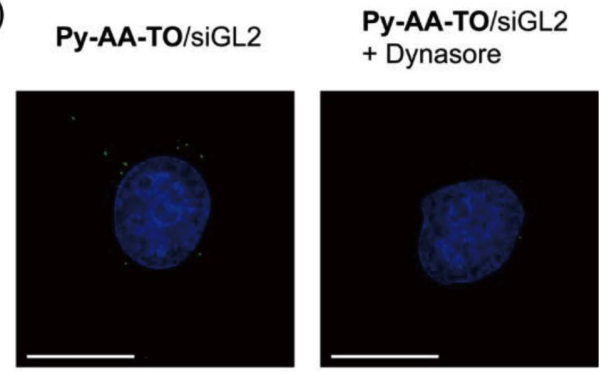

(B)

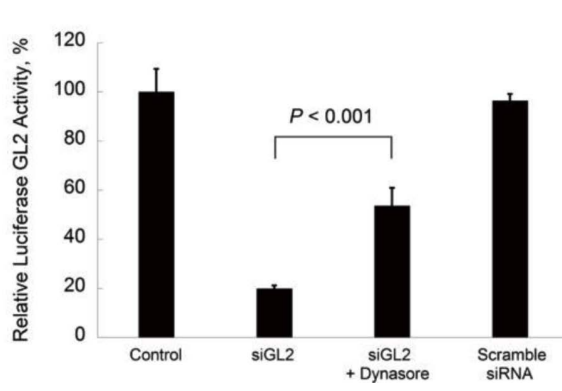

Fig. 2 (A) Fluorescence imaging of living HeLa cells treated with Py-AA-TO/siGL2-containing polyplexes in the absence (left) or presence (right) of $80 \mu \mathrm{M}$ Dynasore. Nuclei were also stained by Hoechst 33342. Images were captured after a $4 \mathrm{~h}$ incubation of the polyplexes. Scale bar: $20 \mu \mathrm{m}$. (B) Effect of Dynasore on RNAi activity of siGL2 against firefly luciferase GL2 gene. Expression level of firefly luciferase GL2 proteins was quantified at $24 \mathrm{~h}$ posttransfection of siGL2-containing polyplexes in the absence or presence of Dynasore. Data were normalized to a buffer control. The result obtained using scramble siRNA is also shown.

a large number of the polyplexes were colocalized with lysosome, yellow spots being seen in the images due to the merging of the green and red colors. ${ }^{17,18,21}$ Based on the Z-series of deconvolved images, ${ }^{17}$ about $80 \%$ of the polyplexes were estimated to be trapped in lysosome (Fig. S1, Supporting Information). From these results, the polyplexes containing siGL2 affnity-labeled with Py-AA-TO were taken up via endocytosis and most of the polyplexes were sequestrated in lysosome, as was typically observed in various kinds of siRNAcontaining polyplexes. ${ }^{22-24}$

We found affinity-labeling with Py-AA-TO was applicable to time-lapse analysis of cellular uptake and subcellular trafficking of the polyplexes (Fig. 4). The bright spots arising from Py-AA-TO/siGL2-containing polyplexes appeared after $20 \mathrm{~min}$ post-transfection. In the course of $2 \mathrm{~h}$, the cellular uptake of the polyplexes gradually proceeded and was almost maximized at $2 \mathrm{~h}$ post-transfection. A large fraction of the intracellular polyplexes was found to be localized in the perinuclear regions, presumably being sequestrated in the acidic vesicles i.e. lateendosome and/or lysosome (cf. Fig. 2) during endocytic pathway. ${ }^{17,18,21,24}$ These kinetics and intracellular behaviors are in good agreement with the results using similar carriers. ${ }^{23-25}$

In our previous study, Py-AA-TO was shown to selectively visualize the siRNA encapsulated in a polymer-based carrier. ${ }^{11}$ We thus examined whether the fluorescence signal of Py-AA-TO could be a good indicator to assess not only cellular uptake but also the disassembly of the polyplex. While the use of two kinds of fluorophores can provide the information of the intracellular disassembly of the polyplex based on fluorescence resonance energy transfer (FRET), ${ }^{26,27}$ such a system requires 

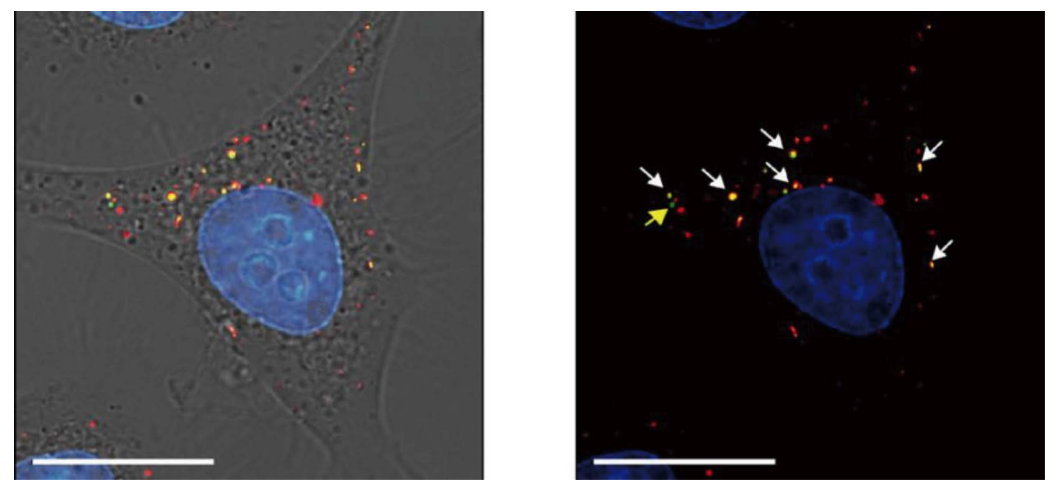

Fig. 3 Images of living HeLa cells treated with Py-AA-TO/siGL2-containing polyplexes in combination with lysosome staining. Lysosome staining was done at $4 \mathrm{~h}$ post-transfection. Nuclei were also stained by Hoechst 33342. The colocalization of polyplexes containing Py-AA-TO/siGL2 and the lysosome-selective fluorescent probe should be detected as yellow spots due to the merging of the green (Py-AA-TO) and red (lysosome-selective fluorescent probe) colors as indicated by white arrows. The fluorescence of the polyplexes which were not merged to that of the lysosome-selective fluorescent probe is indicated by yellow arrows. Scale bar: $20 \mu \mathrm{m}$.
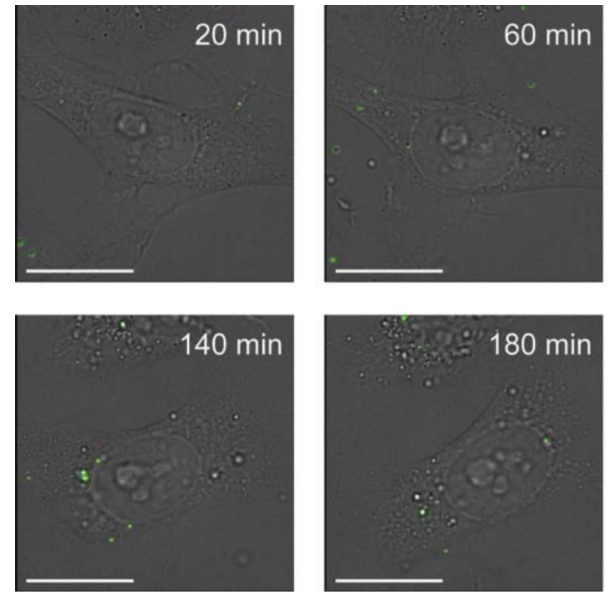
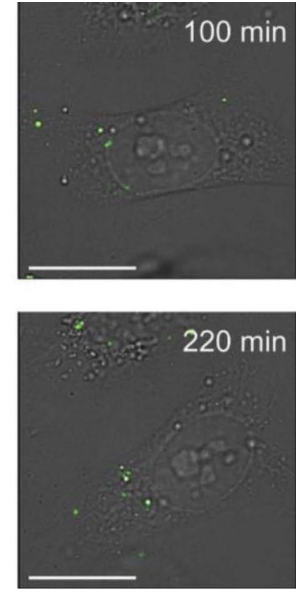

Fig. 4 Time-lapse imaging of Py-AA-TO/siGL2-containing polyplexes during cellular uptake in living HeLa cells. Fluorescence images were captured at indicated time points in the images. Scale bar: $20 \mu \mathrm{m}$.

covalent labeling of fluorophores to siRNAs. To address this, flow cytometric measurements were performed for the cells which were transfected with Py-AA-TO/siGL2-containing polyplexes. Here, we examined the percentage of fluorescent cells at various time points after addition of the polyplexes for the evaluation of cellular uptake and the disassembly of the polyplexes (Fig. 5). For a comparison, we also examined control cells treated with Alexa647-siGL2-containing polyplexes. We found that $76 \%$ of the cells showed fluorescence from Py-AA-TO after a $1 \mathrm{~h}$ incubation with Py-AA-TO/siGL2containing polyplexes. Since it is highly likely that most of the polyplexes were intact at the early stage of cellular uptake (Fig. S2, Supporting Information), ${ }^{25}$ the observed value would correspond to the percentage of the cells that took up the polyplexes. Actually, this value was almost comparable to that of the results using the cells transfected with Alexa647-siGL2containing polyplexes (83\%). Thus, the analysis using Py-AA-TO is applicable to quantification of cellular uptake of the polyplex, as usually performed using fluorophore-labeled siRNA. ${ }^{17,18,21,28}$ Importantly, we found a drastic decrease in the

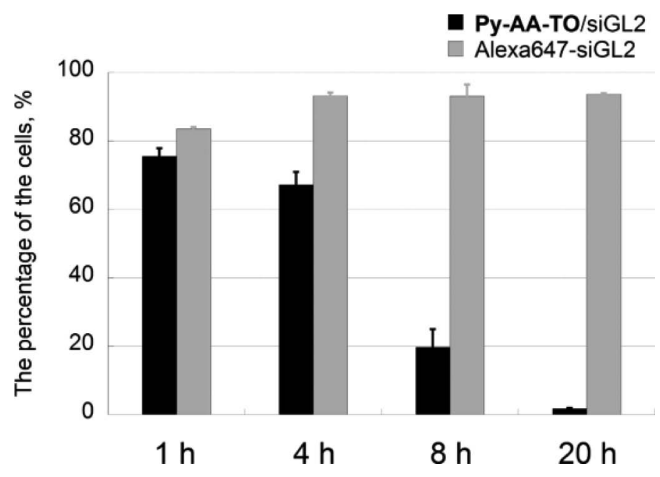

Fig. 5 Flow cytometric analysis of HeLa cells treated with Py-AATO/siGL2 (black) or Alexa647-siGL2 (gray)-containing polyplexes. The percentages of cells that exhibited fluorescence at various time points indicated in the figure are shown. Error bars are standard deviations obtained from three independent experiments. 
percentage of the fluorescent cells treated with Py-AA-TO/ siGL2-containing polyplexes as incubation time increases (Fig. 5). The observed reduction can be interpreted as disappearance of Py-AA-TO fluorescence due to the dissociation of Py-AA-TO from the siRNAs by the disassembly of the polyplexes since Py-AA-TO has modest binding affinity to dTdT overhangs in the siRNAs with the dissociation constant of $3.5 \mu \mathrm{M} .{ }^{11}$ This hypothesis is further supported by an in vitro experiment to examine competition between siRNA in the polyplex and polyanionic heparin. ${ }^{23,29}$ The fluorescence intensity of Py-AA-TO in Py-AA-TO/siGL2-containing polyplexes decreased by addition of heparin in a concentration-dependent manner (Fig. 3, Supporting Information). This indicates the polyplex disassembly induced by heparin releases Py-AA-TO from siRNAs, resulting in reduction of Py-AA-TO fluorescence. It is noteworthy that the polymer-based carrier used here has a similar intracellular half-life of $1-2 \mathrm{~h}$ to that of analogous carriers. ${ }^{26,27}$ In stark contrast, the percentage of the fluorescent cells that took up Alexa647-siGL2-containing polyplexes remained unchanged $(\sim 93 \%)$ even at long incubation time, where siRNA should be released upon disassembly of the polyplexes. This is highly likely due to little change in the fluorescence intensity of Alexa647 covalently labeled with siGL2 whether Alexa647-siGL2 is encapsulated in the carriers or diffused in the cytoplasm. Accordingly, affinity-labeling of siRNAs with Py-AA-TO enables us to analyze the disassembly of the polyplex by monitoring Py-AA-TO fluorescence. This is useful for examination of intracellular half-lives of polymerbased carriers since this parameter can be used for the rational carrier design such as biodegradable polymers to enhance endosomal escape as well as siRNA release in endocytosismediated delivery systems..$^{30-32}$

\section{Conclusions}

In summary, we described the detailed ability of Py-AA-TO as a fluorescent probe for the analysis of the siRNA delivery process. The affinity-labeling of target siRNAs with Py-AA-TO was found to show little influence on the size and zeta potential of the polyplexes. Fluorescence microscopy and flow cytometric measurements revealed that polyplexes containing Py-AA-TO/ siRNA complex were taken up by living HeLa cells through endocytosis and most of them were subsequently sequestrated into lysosome. In contrast to conventional methods using fluorophore-labeled siRNA, our method can be applicable to the analysis of not only cellular uptake but also the intracellular disassembly of the polyplexes by monitoring of Py-AA-TO fluorescence due to its capability of selective visualization of target natural siRNAs encapsulated in the polyplexes. The use of Py-AA-TO thus allows for easy and quantitative analysis of the natural siRNA delivery process, which is essential for the design of polymer-based carriers for the practical implementation of siRNA therapeutics targeting various cancers and diseases.

\section{Acknowledgements}

We thank Prof. Hidetoshi Oikawa and Dr. Tsunenobu Onodera for measurements of physicochemical parameters, and Prof. Tomokazu Matsue and Dr. Hitoshi Shiku for flow cytometric experiments. This work was supported by Grants-in-Aid for Scientific Research (B) (No. 24350033), Challenging Exploratory Research (No. 25620102) from the Ministry of Education, Culture, Sports, Science and Technology, Japan, and by a grant from the Yamada Science Foundation.

\section{Supporting Information}

Lysosomal trap of polyplexes (Fig. S1), fluorescence images of time-lapse experiments (Fig. S2), and fluorescence intensity of polyplexes in the presence of heparin (Fig. S3). This material is available free of charge on the Web at http://www.jsac.or.jp/ analsci/.

\section{References}

1. S. M. Elbashir, J. Harborth, W. Lendeckel, A. Yalcin, K. Weber, and T. Tuschl, Nature, 2001, 411, 494.

2. H. Shen, T. Sun, and M. Ferrari, Cancer Gene Ther, 2012, 19, 367.

3. M. E. Davis, J. E. Zuckerman, C. H. J. Choi, D. Seligson, A. Tolcher, C. A. Alabi, Y. Yen, J. D. Heidel, and A. Ribas, Nature, 2010, 464, 1067.

4. A. Okumura, P. M. Pitha, and R. N. Harty, Proc. Natl. Acad. Sci. U. S. A., 2008, 105, 3974.

5. D. V. Morrissey, J. A. Lockridge, L. Shaw, K. Blanchard, K. Jensen, W. Breen, K. Hartsough, L. Machemer, S. Radka, V. Jadhav, N. Vaish, S. Zinnen, C. Vargeese, K. Bowman, C. S. Shaffer, L. B. Jeffs, A. Judge, I. MacLachlan, and B. Polisky, Nat. Biotechnol., 2005, 23, 1002.

6. A. Gallas, C. Alexander, M. C. Davies, S. Puri, and S. Allen, Chem. Soc. Rev., 2013, 42, 7983.

7. C. V. Pecot, G. A. Calin, R. L. Coleman, G. LopezBerestein, and A. K. Sood, Nat. Rev. Cancer, 2011, 11, 59.

8. P. Resnier, T. Montier, V. Mathieu, J.-P. Benoit, and C. Passirani, Biomaterials, 2013, 34, 6429.

9. C. H. Jones, C.-K. Chen, A. Ravikrishnan, S. Rane, and B. A. Pfeifer, Mol. Pharmaceutics, 2013, 10, 4082.

10. J. C. Burnett and J. J. Rossi, Chem. Biol., 2012, 19, 60.

11. T. Sato, Y. Sato, K. Iwai, S. Kuge, S. Nishizawa, and N. Teramae, Chem. Commun., 2015, 51, 1421.

12. Y.-L. Chiu and T. M. Rana, Mol. Cell., 2002, 10, 549.

13. M. M. Frigault, J. Lacoste, J. L. Swift, and C. M. Brown, J. Cell. Sci., 2009, 122, 753.

14. J. Rejman, V. Oberle, I. S. Zuhorn, and D. Hoekstra, Biochem. J., 2004, 377, 159.

15. A. Ziegler and J. Seelig, Biophys. J., 2008, 94, 2142.

16. O. Boussif, F. Lezoualc'h, M. A. Zanta, M. D. Mergny, D. Scherman, B. Demeneix, and J.-P. Behr, Proc. Natl. Acad. Sci. U. S. A., 1995, 92, 7297.

17. H. Akita, K. Kogure, R. Moriguchi, Y. Nakamura, T. Higashi, T. Nakamura, S. Serada, M. Fujimoto, T. Naka, S. Futaki, and H. Harashima, J. Controlled Release, 2010, 143,311 .

18. A. Tamura, M. Oishi, and Y. Nagasaki, Biomacromolecules, 2009, 10, 1818.

19. E. Macia, M. Ehrlich, R. Massol, E. Boucrot, C. Brunner, and T. Kirchhausen, Dev. Cell, 2006, 10, 839.

20. P. M. McLendon, K. M. Fichter, and T. M. Reineke, Mol. Pharmaceutics, 2010, 7, 738.

21. Y. Sakurai, H. Hatakeyama, Y. Sato, H. Akita, K. Takayama, S. Kobayashi, S. Futaki, and H. Harashima, Biomaterials, 2011, 32, 5733.

22. M. Breunig, U. Lungwitz, R. Liebl, C. Fontanari, J. Klar, A. Kurtz, T. Blunk, and A. Goepferich, J. Gene Med., 2005, 7, 1287.

23. X.-B. Xiong, H. Uludag, and A. Lavasanifar, Biomaterials, 
2009, 30, 242.

24. P. Holzerny, B. Ajdini, W. Heusermann, K. Bruno, M. Schuleit, L. Meinel, and M. Keller, J. Controlled Release, 2012, 157, 297.

25. Z. ur Rehman, D. Hoekstra, and I. S. Zuhorn, ACS Nano, 2013, 7, 3767.

26. C. A. Alabi, K. T. Love, G. Sahay, T. Stutzman, W. T. Young, R. Langer, and D. G. Anderson, ACS Nano, 2012, 6, 6133.

27. H. Lee, I.-K. Kim, and T. G. Park, Bioconjugate Chem., 2010, 21, 289.
28. P. Vader, L. J. van der Aa, J. F. J. Engbersen, G. Storm, and R. M. Schiffelers, J. Controlled Release, 2010, 148, 106.

29. A. Kwok and S. L. Hart, Nanomedicine, 2011, 7, 210.

30. M. Breunig, U. Lungwitz, R. Liebl, and A. Goepferich, Proc. Natl. Acad. Sci. U. S. A., 2007, 104, 14454.

31. K. Buyens, M. Meyer, E. Wagner, J. Demeester, S. C. De Smedt, and N. N. Sanders, J. Controlled Release, 2010, 141,38 .

32. T. F. Martens, K. Remaut, J. Demeester, S. C. De Smedt, and K. Braeckmans, Nano Today, 2014, 9, 344. 